\title{
1. The dominance of the IT industry in a converging ICT ecosystem
}

\section{Anders Henten and Reza Tadayoni}

\section{INTRODUCTION}

The aim of this chapter is to examine the increasing dominance of IT companies in the converging ICT industry and, on the basis of this development, to contribute to extending the theoretical understanding of market and industry convergence in the ICT area. Convergence is one of these concepts that will not go away. The relevance of the concept has been put into question time and again (Lind, 2004). However, convergence developments are increasingly affecting developments within the IT, telecoms and media sectors.

At a technical level as well as at a business and market level, IT, telecoms and media have increasingly been converging during the past decades based technologically on digitisation, in a broad sense, and on Internet, more specifically. In the last decade, it has become still clearer that IT is developing into being the dominant business area in this convergence. Relating to telecoms, the major problems of Nokia in the mobile market are an example of this development, where IT-based companies like Apple and Google presently are prominent players in the smartphone market. With respect to broadcasting, the progress of Over-The-Top (OTT) TV is a recent example of a similar development with IT rising as a dominant field in the convergence processes. Public service broadcasters are trying to counter the increasingly prominent position of IT-based companies in the broadcast area, for instance, with HBBTV (Hybrid Broadband Broadcast TV) initiatives; but they are on the defensive.

The term convergence is often used, but relatively little theory concerning convergence has been developed. At best, definitions of the concept have been suggested and the interrelationships between technical convergence and company, market and industry convergences, and policy and regulatory convergences have been discussed (Saxtoft, 2008). Furthermore the convergence of genres and the implications of the technology and market convergences for content production have been analysed from 
media research points of views (Jenkins, 2006). In the present chapter, emphasis is on industry and market aspects with technology developments as important drivers of convergence.

Greenstein and Khanna (1997) differentiated between convergence based on substitutes and convergence based on complements. Pennings and Puranam (2001) extended this framework of analysis with a supply and demand dimension, establishing a $2 \times 2$ framework, where Stieglitz (2003) later re-labelled supply (technology) and demand (product). These contributions still constitute the basic theoretical understanding of industrial convergence. The theory aim of our chapter is to further extend this framework towards being able to explain why and how new dominance relations develop in converging industries.

The approach used in the chapter is to confront the existing theoretical framework with recent developments in the ICT area. We are not witnessing a convergence on 'equal terms', but a convergence where one of the sectors clearly dominates and sets the conditions for the processes of convergence. This poses the question as to what the mechanisms for such an 'unequal' convergence are and how dominance is structured. An examination of these issues will allow for an extension of the existing theory on industry convergence.

The empirical material discussed is primarily the two exemplary cases of, respectively, the smartphone development, with Nokia as the big loser and the IT companies Apple and Google as big winners, and the video and TV development, with OTT companies on the way forward and traditional TV broadcasters on the defensive.

In order to analyse convergence from a point of view concerning the interplay between technology and market developments, the technology and market aspects will first be presented separately. In the section following the introduction, there is a presentation of technology developments, where emphasis is on the implications of the location of intelligence in the networks at the edges with the Internet end-to-end form of communication. The telecommunication operators have traditionally placed the intelligence inside the networks and have thus been in control of the streams of communication. This has changed with the Internet and the implications of this have become all the more visible with the increasing importance of the Internet.

In the subsequent section, theories regarding industry and market convergences and a theoretical approach to the dominance of one type of player or element vis-à-vis the others in a converging environment are presented. Theories regarding industry and market convergence take their point of departure in the abovementioned publications, primarily the one by Greenstein and Khanna (1997). The theoretical approach concerning 
the dominance relationship is based on the theory of the resource base of companies (Barney et al., 2011), on the one hand, and on the theory of articulation (Barthes, 1972), on the other.

The following two sections examine the cases of, respectively, Nokia vs Apple and Android-based producers in the smartphone markets, and OTT vs HBBTV in the video markets. Finally there is a summary and conclusion extending the theoretical understanding of convergence processes regarding IT, telecoms and media.

\section{TECHNOLOGY DEVELOPMENTS}

The emergence of the Internet is the most radical innovation in the communication field in the last decades. From a technological point of view, this innovation is driven primarily by the digitisation of communication technologies and platforms. The Internet is a 'network of networks' based on the TCP/IP protocol and is designed in a way that enables a radically different environment for innovation, development and organisation of services/applications and infrastructure platforms as compared to telecommunication networks. The history of the Internet, its design principles and the differences between the Internet and regular telecommunication networks are discussed in a number of papers including Saltzer et al. (1981), Clark (1988), Doyle et al. (2002), Willinger and Doyle (2002) and Leiner et al. (2009).

The Internet is engineered in such a manner that it has the potential to integrate all communication services in one and the same network. Some of the key people behind the design of the Internet were asked to write its brief historical account. They start their account by stating: 'The Internet is at once a worldwide broadcasting capability, a mechanism for information dissemination, and a medium for collaboration and interaction between individuals and their computers without regard for geographic location' (Leiner et al., 2009). This has proven to be a true statement, but only when certain conditions are fulfilled - as discussed further in the present section of the chapter. It is important to emphasise that when these conditions are fulfilled, the Internet is the only existing infrastructure that has the ability to integrate all communication services. This is an important issue in our discussion in this chapter concerning the dominance in the convergence process.

A number of design issues in the original development of the Internet provide the Internet with a comparative advantage over other infrastructures (broadcast and telecoms) and thereby give the IT sector a comparative advantage over the media and telecommunication sectors. 
Clark (1988) divided the design principles (goals) into two categories: 1) the fundamental goals regarding the interconnection of different networks ('network of networks') using packet-based technologies; and 2) the 'second level goals', which are defined as a) robustness, b) ability to deliver multiple types of service, c) ability to accommodate a variety of networks, d) distributed architecture, e) cost efficiency, f) host connection with low efforts and g) accountability. Other important parameters that have been crucial for the development of the Internet have been scalability and flexibility, i.e. adaptation to the explosive growth and a design that can and actually does evolve over time in response to changing conditions (Willinger and Doyle, 2002). These design principles have resulted in an 'end-to-end' architecture, where the intelligence is moved from the core to the edges of the network.

Furthermore, the separation of the underlying network technology and the services has been vital in order to remove entry barriers for the service providers when entering the network. The only precondition for service provision is access to the network. This has created huge dynamics in service developments on the Internet. Denton (1999, p. 3) stated that ' $[\mathrm{t}] \mathrm{he}$ end-to end Internet model promises to do away with the idea that anyone would have a monopoly in the definition of services. If the Internet openarchitecture model prevails, a telephone company of the future will still be able to define services, but so will every other user of the communication system capable of writing good code.'

One can say that packet-based switching, the robustness criteria implying a distributed architecture, and the service and network heterogeneity requirements (i.e. being able to deliver multiple types of service and being able to connect multiple networks without requiring changes in their design) were some of the design parameters that enabled the Internet to be a valid candidate to integrate all the communication services in one and the same network. However, when it comes to services that require specific quality of service (QoS) parameters, there are challenges that have worked against this integration.

The problems with QoS stem from the fact that the Internet is based on a 'best effort' principle, which means that the network, in its original design, does not guaranty a timely, reliable or secure communication service. Saltzer et al. (1981) analysed the security issue and argue that designing a network with high levels of security is a waste of resources as many services do not require high levels of security, and that the security issue can be dealt with at the edges of the network for those services that require security. Also the reliability issues are taken care of at the edges using the TCP transport protocol by retransmitting the 'lost packages'. Furthermore, the congestion control mechanism of TCP helps regulate 
from 'the edges' the use of resources in the core of the network in a sophisticated manner (e.g. Schiller, 2003), which dramatically reduces the need for traffic control and management in the core network.

The 'end-to-end argument' also applies to other QoS parameters. The end-to-end architecture enables the applications at the edges of the network to deal with the QoS issues. As the technology develops we can see more and more efficient ways of dealing with QoS parameters at the application level, which supports the provision of 'multiple types of service' on the Internet. 'The layered model of the Internet allows for many applications, each of which would have its own quality of services', suggested Denton (1999, p. 3). There are, however, issues related, in particular, to real time services (like voice and video telephony) that are highly sensitive to end-to-end delays and delay variations and services with high capacity demand (like video/TV) that have had a difficult time being delivered over the Internet. This is why we see that dedicated networks for telephony and TV are still coexisting with the Internet. This situation is, nevertheless, changing because issues related to, for example, delay variation are dealt with at the edge of the network and because the bandwidth capacity/throughput is increasing in the broadband networks.

The real integration of voice/video telephony and TV/video on the Internet is only possible when the development of broadband infrastructures and coding/compression technologies is at a level that makes it possible to deliver these services at the demanded quality over the Internet. As the capacity/throughput is a limited and costly resource, even when the access networks are capable of carrying these services, it has been important for TV/video to implement content delivery networks (CDNs) in the core network to bring the content as close to the users as possible. The CDNs have been instrumental for delivering TV/video over the Internet using different mechanisms including OTT provisions. In recent years, the broadband infrastructures are developing rapidly and CDNs are finding their way into many networks in the advanced markets. This creates good possibilities for the delivery of TV services like Netflix and the like over the Internet. However, in the short- and middle-term perspectives, we will still have dedicated networks for TV as it takes time to deploy broadband networks that can deliver the capacities needed for Ultra HDTV or 3D TV and the like.

As we can see in this brief account, the Internet is based on design principles that make it the prime candidate for integrating all communication services from the outset. The convergence process has in reality been dominated by a process of delivery of more and more communication services over the Internet. The process has been slow because of other circumstances, in particular the fact that broadband networks must be in 
place. But the tendency is clear: All services have shown the potential to be delivered over the Internet or the managed IP networks. In the discussion of the dominance in the convergence process, it becomes clearer why the IT/Internet sector dominates this process. In a geopolitical context, it also becomes clearer that the IT sector in the US has comparative advantages over anyone else in the world owing to its massive involvement in Internet design and development. This also applies to CDNs, which are highly US dominated.

\section{THEORIES ON CONVERGENCE, ARTICULATION AND THE RESOURCE BASE OF COMPANIES}

A large number of publications have been written on convergence from a technology point of view and from market, company, content or regulatory points of views - or combinations thereof (Saxtoft, 2008). However, the issue of convergence itself is relatively little theorised (Lind, 2004; Weaver, 2007). The interplay between technology, market, policy and cultural developments is often put forward as an overall analytical framework. Such an analytical framework provides a good general starting point for understanding the forces promoting convergence at a macro level. However, it does not, for instance, contribute much to the conception of the strategic action possibilities of companies at a micro level, nor does it in any sufficient manner provide a framework for understanding the dominance relationships at the meso and micro levels that we are interested in here.

\subsection{Substitutes and Complements}

Greenstein and Khanna's 1997 paper along with others in the same edited volume was a basic and successful attempt at putting the convergence concept into a theoretical context allowing for a deeper understanding of the business implications of convergence developments. The theoretical framework that Greenstein and Khanna (1997) presented differentiates between convergence based on substitution and convergence based on complementation. If, for instance, one service substitutes for another based on technology convergence - say VoIP substitutes for POT (plain old telephony) - this is a convergence based on substitutes. If, on the other hand, different services are provided on the same network, where they formerly would have been delivered on separate networks, we are dealing with a convergence based on complementation.

However, as pointed out by Greenstein (1999) in a paper summarising 
and discussing the findings of the 1997 Greenstein and Khanna paper, there is often substitution and complementation at the same time. The VoIP service mentioned will, for example, often be delivered as part of a multi-play service package in connection with IPTV and general Internet access. It is also possible that convergence processes will lead to a combination of substitution and complementation resulting in distinctly new services. Herzhoff (2009) found that there are five archetypes of convergence conceptualisation in the information systems (IS) literature: alignment, interoperability, optimisation, recombination and correspondence. Although these conceptualisations emanate from the IS research tradition and not from the business and management research tradition in which the Greenstein and Khanna paper is positioned, there is a considerable degree of overlap between the conceptualisations of convergence, but also inspiration to be found in the archetypes from the IS research tradition.

Pennings and Puranam (2001) extended the substitution/ complementation framework with a supply and demand dimension, constructing a $2 \times 2$ framework of analysis. A substitution-based convergence, for instance, may be initiated from the demand side when 'the needs of different consumer sets become similar' and a complementationbased convergence may take its point of departure in 'different technologies brought together to create new kinds of technology' (Pennings and Puranam, 2001). Furthermore, Stieglitz (2003) re-labels the supply side as the technology variable and the demand side as the product variable in order to specify the primary factors affecting convergence developments.

Other contributions have been made, e.g. Mueller (1999), Lei (2000) and Hacklin et al. (2004). However, the contributions of Greenstein and Khanna (1997), Pennings and Puranam (2001) and Stieglitz (2003) still constitute the basic theoretical point of departure for understanding industrial convergence. Based on the original work by Rosenberg (1963) on the convergence developments in the machine tool industry from 1840 to 1910 , another issue in the convergence literature is concerned with the differentiation between upstream technology convergence and downstream industry convergence (e.g. Gambardella and Torrisi, 1998). Moreover, the issue of convergence versus divergence plays a prominent role in the convergence literature, as industry developments point at the two opposite directions as the 'two sides of a coin' in dynamic industry developments caused by boundary changes in industries (e.g. Christensen, 2010).

In the present chapter, however, there is emphasis on the differentiation between substitution and complementation, as this most precisely points at the issue of dominance relationships between IT, telecoms and media, 
where these different sectors are distinct but yet converge as complements to a large extent but also, to some extent, as substitutes.

\subsection{Articulation}

The articulation concept has several different denotations. In the present paper there is focus on a conceptualisation that is not often seen presently and which takes its point of departure in the application of the concept of articulation by Barthes (1972). Articulation is an important concept in structuralist theory, where it is used to explain how different units are related to one another. Barthes committed a large part of his work to linguistics and semiotics and used the term articulation to signify an activity in which the rules of association between units in texts are discovered and established. Barthes (1972) identified two basic activities in structuralism, dissection and articulation where dissection means the finding of fragments engendering a certain meaning, and articulation means the activity of establishing the relationships between these fragments.

These ideas were taken up by Althusser and Balibar (1969) in their structuralist inspired interpretation of Marxist theory. Balibar introduced the notion that a 'social formation' is an articulation of different 'modes of production' and this notion was further developed by, for instance, Rey (1973) and a long range of other social scientists in the 1970s and 1980s (e.g. Taylor, 1979; Hindess and Hirst, 1977). The idea is that in specific societies, i.e. 'social formations', there can be different 'modes of production' existing at the same time, where 'modes of production' means the way in which goods and services are produced including the basic economic and social relations between classes in society. The modes of production are articulated in specific manners in relation to each other so that they will not only coexist but also be subject to complex interrelationships, where one mode of production will dominate over the others.

Henten and Tadayoni (2002) attempted a transposition of the concept of articulation for the purpose of analysing the interrelationships between traditional broadcasting and video on the Internet. At that point in time, video via Internet was only in its infancy. However, since then IPTV has developed and spread to a much larger extent and OTT TV has developed strongly. Therefore it seems timely to take up the discussion once again on a much broader basis encompassing the whole convergence process of IT, telecoms and media. In addition to the concept of articulation, the dominance relationships emphasised by the use of the concept in relation to the interplay between different modes of production are important to include when analysing the relationships between the fields of IT, telecoms 
and media. Not only do these fields converge; there are also relationships involving dominance between them.

This constitutes an add-on to the idea of convergence as complementation or substitution or combinations thereof. The fields of IT, telecoms and media complement and substitute for each other, subject to conditions of dominance. Other theoretical concepts such as business ecosystems (Moore, 1993) could also be helpful as analytical frameworks for examining the convergence developments. However, the ecosystem concept is better for studying relationships between leading companies - whether 'keystones' or 'dominators' as denoted by Iansiti and Levien (2004) - and the partners with whom they cooperate in smaller scale industrial settings. Specific ecosystems will be the results of certain articulations of IT, telecoms and media. However, for examining the broader picture of the convergence of IT, telecoms and media, the combined concepts of articulation and dominance are seen as a superior framework for analysis.

\subsection{The Resource-based View}

The next step to discuss is why and how the specific conditions of dominance develop. The question that needs to be answered is why it is that companies coming from the telecoms tradition or the media side in the convergence processes do not seem to be able to obtain a dominant position in the convergence of IT, telecoms and media. Many attempts have been made - some of them with success, for instance the i-mode service of NTT DOCOMO - however, most of the attempts with little success. The general picture is that convergence developments increasingly lead in the direction of IT as the dominant field in the convergence of IT, telecoms and media. The question is why companies from telecoms and media do not, to a sufficient degree, move into the IT field and subsume the IT field under their mode of operation.

An important and basic starting point for this discussion is the technology development as presented in the second section of the paper on Internet as an end-to-end network. This development constitutes the technological contribution to the dominance of IT companies in their articulation with companies from the telecoms or media sectors. However, this technological contribution is not sufficient for understanding the dominance of IT in the ICT convergence. Industries and companies are far more than their basic technological platforms. They also encompass the broader sets of resources and capabilities that industries and companies have, including knowledge, labour power, managerial competences and traditions, etc., and they also comprise the roles and positions that these industries and companies have acquired during their lifespan and 
development. This is where the resource-based view (RBV) of companies comes in as a useful framework - or rather the 'dark side' of RBV - as the resources that, in certain contexts, have been the basis for competitive advantages become a barrier in new market contexts. This is a phenomenon that, for instance, Christensen (1997) with the theory of disruptive innovations has pointed at.

The overall aim of the theoretical contributions on the resource base of companies is to determine why and how it is that some companies are able to obtain sustainable competitive advantages as compared to other companies in the same industry. As opposed to the theory of disruptive innovations, RBV focuses on the resources and capabilities (Amit and Schoemaker, 1993) of companies, allowing them to maintain competitive advantages. RBV focuses on the success stories and why and how companies obtain incumbent positions in industries, while disruptive innovations focus on the failures of incumbents.

The fundamental contribution of RBV to the understanding of the position of companies in markets is the focus on the resources that companies have and develop instead of the traditional neoclassical focus on the products - their qualities and prices. The emphasis of RBV is thus on the internal resources and processes in the companies, while neoclassical theory focuses on the external environment - the markets in which the products are sold. According to RBV, the competitive advantage of companies is based on heterogeneous bundles of tangible and intangible assets (Hewitt-Dundas, 2006) comprising knowledge assets, human assets, technical assets, economic assets, etc.

In a summary of the developments of RBV, Barney et al. (2011) provided an overview of the most important contributions to the theory of the resource base of companies and the discussions and criticism of the theory, beginning with Penrose (1959), who presented a starting point for the later contributions on RBV, giving emphasis to the constraints that inadequate resources can put on the development of companies. The term 'resource-based view' was coined by Wernerfelt (1984), and in 1991 Barney developed the characteristics of resources that would provide companies with competitive advantages: The resources must be valuable, rare, inimitable and non-substitutable. Amit and Schoemaker (1993) differentiated between resources and capabilities, where the resources are the companies' assets, and the capabilities are the competences of the companies to leverage those resources. Additionally, Teece, Pisano and Shuen (1997) introduced the idea of dynamic capabilities.

These contributions mostly emphasise the resources and capabilities of companies leading to competitive advantages, except for Penrose (1959), giving emphasis to constraints. The question could then be why it is that 
companies are not able to further develop their 'dynamic capabilities' and why it is that they lose their leading positions, as industries and technologies develop. This is where the theory on disruptive innovations provides some explanatory power (Christensen, 1997). The theory on disruptive innovations builds on the idea of the centrality of the resources and capabilities of companies, but focuses on why it is that companies fail in spite of their former dominant position. The explanation is, in a very brief version, that the resources and capabilities of incumbent companies are too path dependent on the hitherto dominant technologies and business models and, therefore, have great difficulties in changing direction when technology and market circumstances change. This is, to a large extent, what is happening with telecoms and media companies in the new convergent environments. The business models of companies in the telecoms area and in the broadcasting and, in broader terms, media areas have existed for a long time and have developed a certain degree of rigidity in their approach to markets.

\section{NOKIA VERSUS APPLE AND GOOGLE}

The Nokia case illustrates clearly the issues related to the dominance relationships in the convergence processes and in particular the dominance of the IT sector, represented in this case by Apple and Google.

From 1998 to 2011, Nokia was the biggest producer of mobile handsets in the world. Nokia reached almost 50 per cent of the worldwide mobile handset market and was successful in economically more developed markets as well as developing economies. There were a number of reasons for Nokia's success. In terms of the handsets and GUI, Nokia developed better GUIs than other handset producers and their GUIs were recognisable from generation to generation. Furthermore, the terminals were highly robust and of high quality.

If we go further back into history and look for reasons why Nokia became a success from the beginning, it was the timing of its decision to change from analogue to digital, which was of great importance: 'In early 1992, there were many who did not really believe in digital technology as analogue technology was very mature and many established companies would stick to analogue. Nokia chose to believe in the digital technology.' This is a statement taken from an interview with one of the former directors of the company. Other circumstances have been vital for this success, among them: the liberalisation of the European telecom market; the European GSM decision; the reorientation of the Finnish economy towards Western Europe after the downfall of the Soviet Union; the early 
liberalisation of the Finnish telecommunication market; the tradition that came from NMT (Nordic Mobile Telephone); a strong engineering culture in Nokia; and successful strategic buyouts.

The question is then why Nokia was not able to follow up on its previous success in the mobile handset market. This question has a demandside as well as a supply-side dimension. On the demand side, there is a range of different issues relating, e.g., to the usability of mobile handsets, where iPhones, when being launched, simply had a superior usability compared to any other smartphone at the time. There is also the branding issue, where Apple has been the leader since the release of the first iPhones and where Nokia has been the loser. Furthermore, there is the issue of the relative ease of switching from one handset to another for the users. The mobile handset market is a very volatile market, which, for instance, Motorola witnessed before Nokia with the great success and downfall of their Razr phones.

On the supply side, two interrelated sets of factors are relevant for answering the question regarding Nokia's problems in the smartphone market. One set of factors has to do with the overall market developments - in this case, the growth of the smartphone market and the increasing dominance of IT companies in the converging ICT markets. This set of factors could be termed the 'external environment' but it is no more external than allowing for the huge influence that Nokia has had on market developments. This is why one has to see the two sets of factors as interrelated. The other set of factors could be considered as internal in the sense that they are centred on the individual company and its resource base - in this case, Nokia Mobile. The main issues relating to the resources of the company is the fact that Nokia, to a large extent, was an engineering driven company and, furthermore, that it is a telecommunication hardware company. Nokia obviously also had marketing functions and design functions, but the basic DNA of the company is its engineering functions. Moreover, it is basically a telecommunication hardware company. Software is clearly extremely important for mobile handset providers - and even more so with smartphones. Developing and implementing operating systems is a crucial aspect of Nokia's development strategy as can be seen in the emphasis on the development of Symbian and, later, the implementation of Microsoft mobile operating software. However, a major element in explaining the problems Nokia is encountering in the smartphone market is likely to be related to the fact that software is not Nokia's core business.

With respect to business model and ecosystem modelling, Nokia misjudged the dominant business models in the smartphone market. Nokia stayed with the idea that it would be the mobile network operators that 
would organise the upcoming mobile Internet markets. This, however, turned out not to be the case. The organisers and dominant players in the mobile Internet and smartphone markets have turned out to be IT companies - Apple and Google. This entails quite different business models and ecosystems, which Nokia was not able to catch up on and consequently the company was forced into a partnership with Microsoft, which ended up in the total acquisition of Nokia Mobile (Nokia's Devices and Services unit) by Microsoft. What went wrong for Nokia was that the company connected its business model/ecosystem closely with telecommunication operators. In short, Nokia delivered handsets to the telecommunication industry, not putting enough emphasis on the fact that the convergence process changes the dynamics in the mobile market. As soon as the mobile terminals became IP enabled, either through the mobile networks or through Wi-Fi or the like, the stakeholders from IT and Internet could enter and change the business radically. This was what happened with the introduction of the iPhone in 2007 - the first version ever with only Wi-Fi.

When Apple came with iPhone in 2007, Nokia had been in the smartphone market a couple of years and the Nokia N95 was a popular smartphone that sold quite well. But what made iPhone and the later Google Android based ecosystems different was that they used the Internet protocol as the network infrastructure and by that took advantage of the separation between services and network infrastructures by moving the service creation and development from the core to the edge and from the mobile operators to the entrepreneurs at the edge of the network. Consequently, they had the possibility to get shares in the value propositions involving millions of producers and billions of consumers.

In February 2014 just before the final acquisition of Nokia Mobile by Microsoft, the company launched a new family of devices named Nokia X, based on a version of Android OS. These devices are based on Nokia's map (HERE) and their services are based on Microsoft services (Skype, Outlook, etc.) so generally the look and feel is like a Microsoft phone. This could be seen as a signal that even when Nokia becomes an integrated part of Microsoft there remains the possibility of using Android OS in parts of Microsoft's mobile device portfolio. In this chapter we don't discuss what could have happened if Nokia had made this decision to use Android earlier in the process of competition between standards for smartphones, but the decision to develop devices based on Android OS shows even more clearly the superiority and dominance of IT companies like Google in the convergence process. 


\section{THE CASE OF TV OVER INTERNET - FOCUS ON OTT}

As discussed in the section concerning the Internet, Internet can be used as the network infrastructure for delivering TV when certain circumstances are in place and certain requirements are met. There are different ways of delivering TV using the Internet Protocol: IPTV (Internet Protocol TV), HBBTV (Hybrid Broadband Broadcast TV) and OTT TV (Over-The-Top TV). As seen from the following, OTT TV illustrates the IT dominance in the convergence process.

IPTV denotes the provision of TV through broadband networks using the Internet Protocol (IP). IPTV can be provided both as managed services and as a best effort service. However, the term IPTV generally denotes the delivery of TV through managed broadband networks. In such a managed provision, the IPTV provider guarantees a specific degree of quality of service (QoS), while in the case of best effort it is offered through the general Internet. There are a number of different IPTV technologies available on the market, and ITU (the International Telecommunication Union) has specific focus on developing standards for IPTV. IPTV as a managed service uses only the Internet Protocol as the delivery mechanism and does not change the traditional broadcasting business model radically.

Recently, however, the TV sector has witnessed changes following two different tracks: 1) a wave of changes integrating broadcast with the Internet world under the heading of Hybrid Broadband Broadcast TV (HBBTV); and 2) a pure Internet-based approach under the heading of Over-The-Top (OTT) TV technologies and platforms.

The idea of HBBTV platforms is to connect smart TVs to broadcast as well as broadband networks, enabling the broadcasters to develop advanced services related to their content. The HBBTV idea is to make it possible for the broadcasters to retain control of the platforms and the associated applications. EBU (the European Broadcasting Union) has been one of the main promoters of this development.

With OTT, the Internet is seen as the generic platform for the provision of broadcast content and the associated advanced services regarding, for instance, the integration with social networking and community services taking advantage of the proven dynamics and huge innovation potentials of the Internet. This track is US centric with companies like Google and Apple the main players. OTT television is a relatively new term that has arisen partly for technological reasons and partly for business and regulatory reasons. The technological part is concerned with the fact that technological solutions have been developed to ensure a higher degree of 
QoS of television/video delivered over the general Internet. This applies, for instance, to the HLS (HTTP Live Streaming) standard promoted by Apple, the WebM technology developed by Google or the Silverlight Smooth Streaming solution by Microsoft.

The recent technological developments have facilitated the development of business approaches, delivering streaming video and audio content over the general Internet in a sufficiently good quality for the users to utilise it as a source of their consumption of video and audio content. Internationally known examples of such business enterprises are Netflix and Hulu. But there are already a large number of such business companies, and in the coming years there will be a huge variety of companies with a narrower, more national focus. These companies are basically content aggregators assembling different kinds of content (movies, series, and other video and audio programs), which are marketed to the users utilising different types of business model: for example, subscription or advertising.

These types of company are often called third party providers, as they are neither content producers nor network providers. They are content aggregators, who provide content packages over the general Internet though some of them will certainly expand into neighbouring areas such as content production. The fact that they are third parties using the general Internet as the means of transportation also touches upon the regulatory reason for the development of the OTT term. The situation is that ISPs that have been providing managed triple play services lose control of content provided over their networks and become mere 'bit pipes' for the OTT providers. Not only do they lose a business opportunity (triple play), but they are also supposed to deliver the network capacity, which is necessary for the Internet users to get access to the OTT services. In the long run, this will require investments in infrastructure facilities, which the network providers are not certain that they can get compensated by their subscribers with decreasing broadband access prices and flat rate subscriptions. The OTT term pinpoints this situation.

The OTT development must be seen in light of the general relationships between companies coming from the IT world versus companies from the broadcasting and telecommunication world. These different spheres are increasingly converging, but it seems that it is the IT world that is increasingly dominant. As seen in the Nokia case this has already, to a large extent, happened in the mobile communication area, where Androids (Google) and iPhones (Apple) have become dominant. In addition, it also seems to be happening in the television and video/audio area - Apple's and Google's interest here is to get into this new realm of the daily life of users and to combine the video services with their other Internet services. Microsoft has been working on being there for a long time with the Xbox 
as an example. But other large IT companies are also moving into this sphere.

\section{SUMMARY AND CONCLUSION}

An important aim of this chapter has been to attract attention to the emerging relations of dominance in the convergence of IT, telecoms and media. In the context of the shifting relations of dominance internationally in the mobile communications area, Henten, Falch and Vandrup (2010) discussed the increasing US dominance in the mobile Internet area as opposed to earlier phases in mobile developments, where Europe dominated the 2G phase with GSM and Japan forged ahead with NTT DOCOMO's i-mode service at the very beginning of the 21 st century. Since the middle of the first decade of the 21st century, companies from the US have increasingly come to the fore, which Henten, Falch and Vandrup (2010) explain as being based on the strong position of the US regarding IT and Internet.

The chapter has examined two kinds of empirical evidence in order to discuss the emerging relations of dominance in the ICT area. First, there was a comparison of the basic technological properties of Internet communications and telecoms. Furthermore two cases have been discussed: one on the smartphone market and one relating to OTT TV. The two cases clearly illustrate the increasing dominance of the IT area in relation to telecoms and broadcasting, respectively.

The two cases illustrate a general development trend. Digitisation is the very basic technological prerequisite for ICT convergence. However, the Internet constitutes the more specific digital infrastructure that today is a fundamental defining basis for the actual convergence developments. How digital convergence would have developed without Internet is, to a large extent, a speculative endeavour, although prior but terminated technology developments could illustrate other development paths. One example of such a prior development path is ATM-based network technologies initiated by the telecoms field, which have lost importance with the proliferation of TCP/IP. Another example is the development of videotex in the late 1980s and early 1990s. This was a system developed by telecoms companies that preceded the graphical interface developed in connection with the Internet World Wide Web. However, with the development of the WWW, videotex quickly disappeared except in France, where it vanished more slowly, as the French incumbent telecoms operator had invested enormously in its videotex system, Teletel (Bouwman and Christoffersen, 1992). 
Internet is thus the core defining technological complex, and although Internet did not come out of private IT companies, IT companies - and especially US-based IT companies - were much faster to incorporate Internet technologies into their products and services. Internet with its end-to-end property was basically in line with the networking interests of IT companies. IT companies in the US had worked since the 1960s on getting into networking, including having the right to use the telecommunication networks for computer networking. What the IT companies needed was for the networks to work as dump bit-pipes for their computer communication and this collided with the interests of the telecommunication operators who wanted to maintain control of the networks, which entailed keeping the intelligence in the networks.

The basic philosophy of the Internet was well in line with the interests of IT companies. Furthermore, IT companies were the new 'kids on the block'. The IT field was the new dynamic business area as opposed to telecommunication and broadcasting, which had existed for approximately 100 years in the case of telecoms and approximately 50 years in the case of broadcasting. This has meant that telecoms and broadcasting are embedded in longstanding modes of operation that have constrained their possibilities for adopting the new Internet mode of operation. Part of this is also that telecoms as well as broadcasting have traditionally been tightly regulated sectors, which adds an additional element to their path dependent behaviour.

The increasing emergence of IT in ICT convergence has thus been on its way for quite some time. However, development of the relationships between IT, telecoms and media has far from ended. It is a continuous process, and the purpose of this chapter has been to analyse how these processes of convergence evolve in terms of complementation, substitution and dominance.

The differentiation between complementation and substitution proposed by Greenstein and Khanna (1997) constitutes a good point of departure for analysing how convergence between IT, telecoms and media evolves in terms of new products and services as a result of novel combinations and the closing down and establishment of other product and service areas. This provides a valuable framework for analysing the strategic options of companies in such markets. However, it does not provide a sufficient framework for understanding the conditions for dominance between the different types of market players. The articulation concept provides the foundation for such a framework. While the concept of articulation has been used for how 'modes of production' are combined, it can also be used for the combination of the modes of operation of different market players, coming from IT, telecoms and media, respectively. 
Additionally, in order to understand the mode of operation of the different types of company and the different kinds of sector, the resource-based view of companies with its emphasis on the resources and capabilities that promote or constrain the development paths of the companies in question becomes the third element in a theoretical framework that helps in analysing the evolving dominance relationships in ICT convergence.

Adding the articulation of modes of operation, the resource-based view and its kindred theory on disruptive innovations makes it possible to examine the relations of dominance in ICT convergence, where there are strong indications that IT is emerging as the dominant field. The manner in which IT, telecoms and media are articulated allows for companies from the IT field to subsume the interest of companies from the telecoms and media fields under their strategic interests. Therefore, the framework proposed is a combination of the complementation/substitution framework with the articulation concept used on modes of operation and the resource-based view on companies extended to a sector perspective and the path dependent developments of the IT, telecoms and media sectors.

\section{REFERENCES}

Althusser, L. and E. Balibar (1969), Lire 'Le Capital', Paris: Maspero.

Amit, R. and P.J.H. Schoemaker (1993), 'Strategic assets and organizational rent', Strategic Management Journal, 14 (1), 33-46.

Barney, J.B. (1991), 'Firm resources and sustained competitive advantage', Journal of Management, 17 (1), 99-120.

Barney, J.B., D.J. Ketchen Jr. and M. Wright (2011), 'The future of resource-based theory: revitalization or decline?', Journal of Management, 37 (5), 1299-1315.

Barthes, R. (1972), 'The structuralist activity', in R. Barthes, Critical Essays, Evanston, IL: Northwestern University Press.

Bouwman, H. and M. Christoffersen (eds) (1992), Relaunching Videotex, Dordrecht: Kluwer Academic Publishers.

Christensen, C.M. (1997), The Innovator's Dilemma: When New Technologies Cause Great Firms to Fail, Boston: Harvard Business School Press.

Christensen, J.F. (2010), Towards a Framework of Industry and Product Market Convergence, DRUID, Imperial College London Business School, 16-18 June 2010, available at http://www2.druid.dk/conferences/viewpaper. php?id=501467\&cf=43 (accessed 11 April 2013).

Clark, D.D. (1988), 'The design philosophy of the DARPA Internet protocols', SIGCOMM '88 Symposium Proceedings on Communications Architectures and Protocols, ACM, New York, pp. 106-114.

Denton, T.M. (1999), Netheads versus Bellheads: Research into Emerging Policy Issues in the Development and Deployment of Internet Protocols, final report for the Federal Department of Industry, Contract Number U4525-9-0038, available at http://www.tmdenton.com/images/papers/bellheads.pdf (accessed 26 February 2015). 
Doyle, J.C., S.H. Low, F. Paganini, G. Vinnicombe, W. Willinger and P. Parrilo (2002), Robustness and the Internet: Theoretical Foundations, available at http:// netlab.caltech.edu/publications/RobustInternetII-Doyle-2002.pdf (accessed 2 May 2013).

Gambardella, A. and S. Torrisi (1998), 'Does technological convergence imply convergence in markets? Evidence from the electronics industry', Research Policy, 27 (5), 445-63.

Greenstein, S. and T. Khanna (1997), 'What does industry convergence mean?', in D.B. Yoffie (ed.), Competing in the Age of Digital Convergence, Boston, MA: Harvard Business School Press, pp. 201-26.

Greenstein, S. (1999), Technological Convergence, mimeo, available at http://www. kellogg.northwestern.edu/faculty/greenstein/images/htm/Research/articles/con vergence-handbook-final.pdf (accessed 2 May 2013).

Hacklin, F., V. Raurich and C. Marxt (2004), 'How incremental innovation becomes disruptive: the case of technology convergence', Proceedings of the IEEE International Engineering Management Conference, Singapore, 2004, available at http://ieeexplore.ieee.org/stamp/stamp.jsp?tp=\&arnumber $=1407070$ (accessed 5 April 2013).

Henten, A. and R. Tadayoni (2002), Constitution of Markets for Internet TV, CTI Working Papers No.71, Lyngby: DTU.

Henten, A., M. Falch and K. Vandrup (2010), 'Mobile Internet developments in Europe, East Asia and the US', in A. Gentzoglanis and A. Henten (eds), Regulation and the Evolution in the Global Telecommunications Industry, Cheltenham: Edward Elgar Publishing, pp. 317-39.

Herzhoff, J. (2009), 'The ICT Convergence Discourse in the Information Systems Literature: A Second-Order Observation', 17th European Conference on Information Systems, 8-10 June 2009, Verona, Italy, available at http://digitalinfrastructures.org/publications/Herzhoff2009.pdf (accessed 28 February 2014).

Hewitt-Dundas, N. (2006), 'Resource and capability constraints to innovation in small and large plants', Small Business Economics, 26, 257-77.

Hindess, B. and P. Hirst (1977), Mode of Production and Social Formation, London: MacMillan.

Iansiti, M. and R. Levien (2004), 'Strategy as ecology', Harvard Business Review, $82(3), 68-78$.

Jenkins, H. (2006), Convergence Culture, New York: New York University Press.

Lei, D.T. (2000), 'Industry evolution and competence development: the imperatives of technological convergence', International Journal of Technology Management, 19 (7/8), 699-738.

Leiner, B.M., V.G. Cerf, D.D. Clark, R.E. Kahn, L. Kleinrock, D.C. Lynch, J. Postel, L.G. Roberts and S. Wolff (2009), Brief History of the Internet, available at http://www.internetsociety.org/internet/what-internet/history-internet/ brief-history-internet (accessed 31 May 2013).

Lind, J. (2004), Convergence: History of Term Usage and Lessons for Firm Strategies, available at http://www.userpage.fu-berlin.de/ jmueller/its/. ../1_ LIND.doc (accessed 20 May 2013).

Moore, J.F. (1993), 'Predators and prey: a new ecology of competition', Harvard Business Review, 71 (2), 75-86.

Mueller, M.L. (1999), 'Digital convergence and its consequences', The Public, 6 (3), 11-28.

Pennings, J.M. and P. Puranam (2001), Market Convergence and Firm Strategy: 
New Directions for Theory and Research, paper presented at the ECIS Conference on 'The Future of Innovation Studies', 20-23 September, Eindhoven, the Netherlands.

Penrose, E. (1959), The Theory of the Growth of the Firm, New York: Wiley.

Rey, P.-P. (1973), Les Alliances des Classes, Paris: Maspero.

Rosenberg, N. (1963), 'Technological change in the machine tool industry, 1840-1910', The Journal of Economic History, 23 (4), 414- 43.

Saltzer, J.H., D.P. Reed and D.D. Clark (1981), 'End-to-end argument in system design', Proceedings of the Second International Conference on Distributed Systems, Paris, France, 8-10 April 1981, IEEE Computer Society, pp. 509-12.

Saxtoft, C. (2008), Convergence: User Experience, Communications Enablers and Business Opportunities, Hoboken, NJ: Wiley.

Schiller, J.H. (2003), Mobile Communications, 2nd edn, Boston, MA: Addison-Wesley.

Stieglitz, N. (2003), 'Digital dynamics and types of industry convergence: the evolution of the handheld computers market in the 1990s and beyond', in J.F. Christensen and P. Maskell (eds), The Industrial Dynamics of the New Digital Economy, Cheltenham, UK and Northampton, MA: Edward Elgar Publishing, Chapter 7.

Taylor, J.G. (1979), From Modernization to Modes of Production: A Critique of the Sociologies of Development and Underdevelopment, London: Macmillan.

Teece, D., G. Pisano and A. Shuen (1997), 'Dynamic capabilities and strategic management', Strategic Management Journal, 18 (7), 509-34.

Weaver, B. (2007), 'Industry convergence: driving forces, factors and consequences', 19th NFF Conference, Bergen, 9-11 August 2007, available at http:// www.nhh.no/Files/Filer/institutter/for/conferences/nff/papers/weaver (accessed 24 May 2013).

Wernerfelt, B. (1984), 'A resource-based view of the firm', Strategic Management Journal, 5 (2), 171-80.

Willinger, W. and J.C. Doyle (2002), Robustness and the Internet: Design and Evolution, available at http://netlab.caltech.edu/publications/JDoylepart1_ vers42002.pdf (accessed 20 March 2013). 